DOI: https://doi.org/10.24297/jam.v19i.8802

\title{
On the existence of continuous solutions of a nonlinear quadratic fractional integral equation
}

\author{
Wagdy G. El-Sayed ${ }^{1}$, Mahmoud M. El-Borai ${ }^{2}$, Mohamed M.A. metwali ${ }^{3}$, Nagwa I. Shemais ${ }^{4}$ \\ ${ }^{1,2}$ Faculty of Science, Alexandria University, Alexandria, Egypt \\ ${ }^{3,4}$ Department of mathematics, Faculty of Science, Damanhour Universty, Egypt \\ ${ }^{1}$ wagdygoma@alexu.edu.eg, ${ }^{2}$ m_m_elborai@yahoo.com, ${ }^{3}$ m.metwali@yahoo.com, ${ }^{4}$ n.ibrahim90@yahoo.com
}

\begin{abstract}
We prove an existence theorem for a nonlinear quadratic integral equation of fractional order, in the Banach space of real functions defined and continuous on closed interval. This equation contains as a special cases numerous integral equation studied by other authors. Finally, we give an example for indicating the natural realizations of our abstract result presented in this paper.
\end{abstract}

Keywords: Quadratic integral equation; measure of noncompactnes; Darbo's fixed point theorem; fractional calculus. Biographical notes: Wagdy G. El-Sayed received his PhD in Integral Equations from Maria Curie - Sklodowiska University in Lublin - Poland. He is currently an Associate Professor and Professor at the Department Mathematics and Computer Science, Faculty of Science, Alexandria University, Alexandria - Egypt. His research is interesting in the Solvability of some different integral equations or functional integral equations or integro-differential equations or quadratic integral equations in different classes of integrable functions, where he try to assume the sufficient conditions under which the existence theorem of a certain integral equation can be proved by using Darbo fixed point theorem via some measures of noncompactness. He published in journals such as Journal of Mathematical Analysis and Applications, Budapest University, Hungary, International Journal of Mathematics, Florida - USA, Journal of Applied Mathematics and Computation

Mahmoud M. El-Borai Editor in this Journal

Mohamed M.A. Metwali obtained his Ph.D. from A. Mickiewicz University, Poznan, Poland in topic of "Differential and integral equations". He is currently working as Associate Professor at Department of Mathematics, Faculty of Science, Damnhour University, Egypt. His research interests are in the areas of Integral Equations, Differential Equations, Function Analysis, set valued Analysis, time scales, fixed point theory, and solutions in different function spaces such as "Lesbegue spaces, Orlicz spaces, space of regulated functions, space of Bounded variation, space of continuous functions". He published many papers in various journals such as J. Math. Anal. Appl., Mediter. J. Math, Electron. J. Qual. Theory of Differ. Equ., Opuscula Math., Math. Slovaca, J. Fixed Point Theory Appl., Carpathian J. Math., Rocky Mountain J. Math., and Demonstratio Mathematica.

Nagwa I. Shemais received her MSc in Dynamical system. She is currently a PhD candidate at the Department of Mathematics, Faculty of Science, Damanhour University, Egypt. Her research interests are in the areas of nonlinear analysis. 


\section{Introduction}

Several problems in many branches of science can be modeled as an integral equation such as in physics, engineering, biology,...ect see for example([4, [6], [7], [14]). Quadratic integral equations have many useful applications in describing problems in the real world. For example, quadratic integral equations are applicable in the kinetic theory of gases, in the theory of neutron transport and in the theory of radiative transfer. Especially the so called quadratic integral equation of Chandrsekher type can be seen in many application(cf.([9], [10] [26], [12, [25]). On the other hand the fractional calculus topic is enjoying growing interest of engineers and scientists, see ([16], [17, [19], [20]) . The most used techniques to study the existence for solutions of an integral equation are based on fixed point argument for example, Banach fixed point theorem was used by many authors in order to establish existence results for different kind of integral equations (see, for example[ [8], 28] ]). But in the absence of compactness and the Lipschitz condition, the above mentioned theorem cannot be applied and in this case, the measure of non-compactness can be useful. Many existence results for integral equations were established using fixed point theorems involving measure of non-compactness. For most details, we refer the reader to the references therein and to([1], [2], [3], [11, [16], [21], [27]). In this paper we deal with quadratic integral equation of fractional order with respect to another function. Using fixed point theorem via measure of non-compactness Due to Darbo are the main tool in carrying out our proof.

\section{Preliminaries}

The main purpose of this paper to study the following integral equation

$$
x(t)=f(t)+g(t, x(t))+\frac{(A x)(t)}{\Gamma(\alpha)} \int_{a}^{t} \frac{m^{\prime}(s) u(t, s, x(s), x(\lambda s))}{(m(t)-m(s))^{1-\alpha}} d s, t \in I=\left[a, T_{1}\right],
$$

where $\alpha \in(0,1), 0 \leq a \leq T_{1}, \quad f: I \rightarrow R, g: I \times R \rightarrow R$, $u: I \times I \times R \times R \rightarrow R$, and $m: I \rightarrow R$, from the view of the theory of existence of solutions. Eq(1) can be written in the form

$$
x(t)=f(t)+g(t, x(t))+(T x)(t) I_{a, m}^{\alpha} u(t, ., x(.), x(.)),
$$

where $I_{a, m}^{\alpha}$ is the fractional integral of order $\alpha$ with respect to the function $m$ defined by

$$
\frac{1}{\Gamma(\alpha)} \int_{a}^{t} \frac{m^{\prime}(s) u(t, s, x(s), x(\lambda s))}{(m(t)-m(s))^{1-\alpha}} d s, \quad \text {. } \quad t \in I=\left[a, T_{1}\right]
$$

which in particular cases we have:

(i) If $m(s)=\ln s$ and $a>0$ then $I_{a, m}^{\alpha}$ is the Hadamard fractional integral,

(ii) If $m(s)=s^{\beta}(\beta>0)$ then $I_{a, m}^{\alpha}$ is the Erdélyi-Kober fractional integral,

(iii) If $m(s)=s,(\beta=1)$ and $a>0$ in such case $I_{a, m}^{\alpha}$ is the Riemann-Liouville fractional integral.

Using a measure of non-compactness in the set $C([a, T] ; R)$ of the real continuous functions in $[a, T]$, we need to recall some basic concepts that will need later. let $E$ be an infinite dimensional Banach space with norm $\|$.$\| and zero element$ $\theta$. The symbols $\bar{X}$ and ConvX stand for the closure and closed convex hull of a subset $X$ of $E$, respectively. Now denote $\mathcal{M}_{E}$ the family of all nonempty and bounded subsets of $E$, and $\mathcal{N}_{E}$ and $\mathcal{N}_{E}^{W}$ denotes the family of all nonempty relatively compact and weakly relatively compact sets, respectively. The symbol $\bar{X}^{W}$ stands for the weak closure of a set $X$ while $\bar{X}$ denotes its closure. 
Definition 2.1 (Measure of noncompactness) [11]

A mapping $\mu: \mathcal{M}_{E} \rightarrow[0, \infty)$ is said to be a measure of noncompactness in $E$ if it satisfies the following conditions:

(1) the family $\operatorname{ker} \mu=\left\{X \in \mathcal{M}_{E}: \mu(X)=0\right\}$ is nonempty and ker $\mu \subset \mathcal{N}_{E}$, where ker $\mu$ is called the kernel of the measure $\mu$.

(2) $X \subset Y \Rightarrow \mu(X) \leq \mu(Y)$.

(3) $\mu(\operatorname{Conv} X)=\mu(X)=\mu(\bar{X})$.

(4) $\mu[\lambda X+(1-\lambda) Y] \leq \lambda \mu(X)+(1-\lambda) \mu(Y), \lambda \in[0,1]$.

(5) If $X_{n} \in \mathcal{M}_{E}, X_{n}=\bar{X}_{n}$ and $X_{n+1} \subset X_{n}$ for $n=1,2, \ldots$ and if

$$
\lim _{n \rightarrow \infty} \mu\left(X_{n}\right)=0 \text {, then } X_{\infty}=\bigcap_{n=1}^{\infty} X_{n} \neq \varphi \quad \text { (nonempty and compact). }
$$

A function $\phi:[0, \infty) \rightarrow[0, \infty)$ is said to be a comparison function if it satisfies the following properties:

(i) $\phi$ is non-decreasing;

(ii) $\lim _{n \rightarrow \infty} \phi^{(n)}(t)=0$ for all $t>0$, where $\phi^{(n)}$ denotes the $n$ - iteration of $\phi$.

Examples of comparison functions are

$$
\phi(t)=\lambda t^{3}, \quad \lambda \in[0,1) ; \quad \phi(t)=\arctan (t)
$$

We denote the set of comparison functions by $\boldsymbol{\Phi}$ as in [15]

Lemma 2.1 [1]: Let $\phi:[0, \infty) \rightarrow[0, \infty)$ be a nondecreasing and upper semi-continuous function. Then the following conditions are equivalent:

(i) $\lim _{n \rightarrow \infty} \phi^{(n)}(t)=0$ for all $t>0$.

(ii) $\phi(t)<t$ for all $t>0$.

Lemma 2.2 ([1], [15]): Let $C$ be a nonempty, bounded, closed and convex subset of Banach space $E$. Let $U: C \rightarrow C$ be a continuous transformation such that

$$
\mu(U W) \leq \phi(\mu(W)), \quad W \subseteq C
$$

Where $\phi \in \mathbf{\Phi}$ and $\mu$ is a measure of non-compactness in E. Then $U$ has at least one fixed point.

For given functions $\phi, \psi:[0,1) \rightarrow \mathbb{R}$ and a real number $\lambda$, define the operator $\mathrm{T}_{\max }$ and $\mathrm{T}$ by

$$
\mathrm{T}_{\max }(\phi, \psi)(t)=\max \{\phi(t), \psi(t)\}, \quad t \geq 0
$$

and

$$
\mathrm{T}(\lambda, \phi)(t)=\lambda \phi(t), \quad t \geq 0
$$

Denote $C([0, \infty) ; \mathbb{R})$ the set of all real continuous functions in $[0, \infty)$ and $\tilde{\boldsymbol{\Phi}}$ by the set $\boldsymbol{\Phi} \cap C([0, \infty) ; \mathbb{R})$. In $([15])$, Sadarangain and Samet proved the following useful result. 
Lemma 2.3 The following properties hold:

(i) $\mathrm{T}_{\max }(\boldsymbol{\Phi} \times \boldsymbol{\Phi}) \subseteq \boldsymbol{\Phi}$.

(ii) $\mathrm{T}([0,1] \times \boldsymbol{\Phi}) \subseteq \boldsymbol{\Phi}$.

Let $E=C\left(\left[a, T_{1}\right] ; \mathbb{R}\right)$ be a Banach space defined on the set $C$ of real continuous functions in $\left[a, T_{1}\right]$ with norm

$$
\|v\|=\max \left\{|v(t)|: t \in\left[a, T_{1}\right]\right\}, \quad v \in C\left(\left[a, T_{1}\right] ; \mathbb{R}\right) .
$$

We will use through this paper the following notations. Let $B \in \mathcal{M}_{E}$. For $v \in B$ and $\epsilon \geq 0$, set

$$
\omega(v, \epsilon)=\sup \left\{|v(t)-v(s)|: t, s \in\left[a, T_{1}\right], \quad|t-s| \leq \epsilon\right\}
$$

We define the mapping $\Omega: \mathcal{M}_{E} \times[0, \infty) \rightarrow[0, \infty)$ by

$$
\Omega(B, \epsilon)=\sup \{\omega(v, \epsilon): v \in B\}, \quad(B, \epsilon) \in \mathcal{M}_{E} \times[0, \infty) \rightarrow[0, \infty)
$$

It was proved in[11] that the mapping $\mu: \mathcal{M}_{E} \rightarrow[0, \infty)$ defined by

$$
\mu(B)=\lim _{\epsilon \rightarrow 0^{+}} \Omega(B, \epsilon), \quad B \in \mathcal{M}_{E}
$$

is a measure of non-compactness in the Banach space $E$. In this section, we will investigate the existence theorem of our integral equation(1) under suitable assumptions:

\section{Main result}

Consider the following assumptions:

$\left(a_{1}\right) \quad f: I \rightarrow \mathbb{R}$; is continuous nondecreasing and nonnegative function on $I$,

$\left(a_{2}\right)$ the function $g: I \times \mathbb{R} \rightarrow \mathbb{R}$ is continuous.

$\left(a_{3}\right)$ There exists a function $\phi_{1}:[0, \infty) \rightarrow[0, \infty)$ such that

(i) $\phi_{1}(0)=0$.

(ii) $\phi_{1}$ is nondecreasing and continuous.

(iii) there exist $\delta \geq 0$ and $\theta \in \tilde{\boldsymbol{\Phi}}$ such that

$$
\phi_{1}(t) \leq \delta \theta(t), \quad t \geq 0
$$

(iv) for all $(t, u, v) \in I \times \mathbb{R} \times \mathbb{R}$ we have

$$
|g(t, u)-g(t, v)| \leq \phi_{1}(|u-v|)
$$

$\left(a_{4}\right)$ The operator $A: C(I) \rightarrow C(I)$ is continuous and satisfies the Darbo condition for measure of non compactness $\mu$ with the constant $q, A$ is positive operator( $A x \geq 0$ if $x \geq 0)$, 
$\left(a_{5}\right)$ There exist nonnegative constants $a$ and $b$ such that

$$
|(A x)(t)| \leq(a+b\|x\|)
$$

for each $x \in C(I)$ and $t \in I$,

$\left(a_{6}\right) \quad u: I \times I \times \mathbb{R} \times \mathbb{R} \rightarrow \mathbb{R}$ is continuous function such that $u: I \times I \times \mathbb{R}_{+} \times \mathbb{R}_{+} \rightarrow \mathbb{R}_{+} u(t, s, x, y)$ is nondecreasing with respect to each variable separately,

$\left(a_{7}\right)$ There exists a nondecreasing function $\phi: \mathbb{R}_{+} \times \mathbb{R}_{+} \rightarrow \mathbb{R}_{+}$s.t

$$
|u(t, s, x, y)| \leq \phi(|x|,|y|) \quad \forall t, s \in \text { Iand } \quad x, y \in \mathbb{R}
$$

$\left(a_{8}\right) m \in C\left(\left[a, T_{1}\right], R\right) \cap C^{1}\left(\left(a, T_{1}\right], R\right)$ with $m^{\prime}(t)>0$ for all $t \in\left(a, T_{1}\right]$,

$\left(a_{9}\right)$ There exists a positive constant $r_{0}$ such that

$$
\|f\|+\phi_{1}\left(r_{0}\right)+g^{*}+\frac{\left(a+b r_{0}\right)}{\Gamma(\alpha+1)} \cdot \phi\left(r_{0}, r_{0}\right)\left(m\left(T_{1}\right)-m(a)\right)^{\alpha} \leq r_{0}
$$

where

$$
g^{*}=\max \left\{|g(t, 0)|: \quad t \in\left[a, T_{1}\right]\right\}
$$

Theorem 3.1 Under the assumptions $\left(a_{1}\right)-\left(a_{9}\right)$, the $\left.E q \mathbb{1}\right)$ has at least one solution $x^{*} \in C\left(\left[a, T_{1}\right] ; \mathbb{R}\right)$. Moreover $\left\|x^{*}\right\| \leq r_{0}$.

Proof. Let us consider the operator $F$ defined on $E=C\left(\left[a, T_{1}\right], R\right)$ by

$$
(F x)(t)=f(t)+g(t, x(t))+\frac{(A x)(t)}{\Gamma(\alpha)} \int_{a}^{t} \frac{m^{\prime}(s) u(t, s, x(s), x(\lambda s))}{(m(t)-m(s))^{1-\alpha}} d s,(x, t) \in E \times\left[a, T_{1}\right] .
$$

At first,we show that the operator $F: C\left(\left[a, T_{1}\right], \mathbb{R}\right) \rightarrow C\left(\left[a, T_{1}\right], \mathbb{R}\right)$, set

$$
(U x)(t)=\frac{1}{\Gamma(\alpha)} \int_{a}^{t} \frac{m^{\prime}(s) u(t, s, x(s), x(\lambda s))}{(m(t)-m(s))^{1-\alpha}} d s, \quad(x, t) \in E \times\left[a, T_{1}\right] .
$$

From assumption $\left(a_{1}\right)\left(a_{2}\right)\left(a_{4}\right)$ We have just to prove $U$ maps $E$ into itself, that is $U x:\left[a, T_{1}\right] \rightarrow \mathbb{R}$ is continuous for every $x \in E$. Observe that $U x$ is well defined for all $t \in\left[a, T_{1}\right]$.

$$
\begin{aligned}
&|(U x)(t)| \leq \frac{1}{\Gamma(\alpha)} \int_{a}^{t} \frac{\left|m^{\prime}(s) \| u(t, s, x(s), x(\lambda s))\right|}{(m(t)-m(s))^{1-\alpha}} d s \\
& \leq \frac{1}{\Gamma(\alpha)} \cdot \phi(\|x\|,|\lambda| \cdot\|x\|) \int_{a}^{t}(m(t)-m(s))^{\alpha-1} d s \\
& \leq \frac{1}{\alpha \Gamma(\alpha)} \cdot \phi(\|x\|,\|x\|)(m(t)-m(a))^{\alpha} \\
& \leq \frac{1}{\Gamma(\alpha+1)} \cdot \phi(\|x\|,\|x\|)\left(m\left(T_{1}\right)-m(a)\right)^{\alpha}<\infty . \\
&|\quad(F x)(t)|=\mid(f(t)\left.+g(t, x(t))+\frac{(A x)(t)}{\Gamma(\alpha)} \int_{a}^{t} \frac{m^{\prime}(s) u(t, s, x(s), x(\lambda s))}{(m(t)-m(s))^{1-\alpha}} d s\right) \mid \\
& \leq \quad|f(t)|+|g(t, x(t))-g(t, 0)|+\frac{|(A x)(t)|}{\Gamma(\alpha)} \int_{a}^{t}\left|\frac{m^{\prime}(s) u(t, s, x(s), x(\lambda s))}{(m(t)-m(s))^{1-\alpha}}\right| d s
\end{aligned}
$$




$$
\begin{aligned}
& \leq|f(t)|+\phi_{1}(|x(t)|)+|g(t, 0)|+\frac{|(A x)(t)|}{\Gamma(\alpha)} \int_{a}^{t}\left|\frac{m^{\prime}(s) u(t, s, x(s), x(\lambda s))}{(m(t)-m(s))^{1-\alpha}}\right| d s \\
& \leq\|f\|+\phi_{1}(\|x\|)+g^{*}+\frac{(a+b\|x\|)}{\Gamma(\alpha)} \int_{a}^{t} \frac{\left|m^{\prime}(s)\right||u(t, s, x(s), x(\lambda s))|}{(m(t)-m(s))^{1-\alpha}} d s \\
& \leq\|f\|+\phi_{1}(\|x\|)+g^{*}+\frac{(a+b\|x\|)}{\Gamma(\alpha+1)} \cdot \phi(\|x\|,\|x\|)(m(T)-m(a))^{\alpha} \\
& <\infty .
\end{aligned}
$$

To show the continuity of $U x$ at the point $a$. Let $\left\{t_{n}\right\}$ be a sequence in $\left[a, T_{1}\right]$ such that $t_{n} \rightarrow a^{+}$as $n \rightarrow \infty$ using the above result, for all $n \in N$, we have

$$
\begin{aligned}
\left|(U x)\left(t_{n}\right)\right| & \leq \frac{1}{\Gamma(\alpha)} \phi(\|x\|,\|x\|) \int_{a}^{t}\left(m\left(t_{n}\right)-m(s)\right)^{\alpha-1} d s \\
& \leq \frac{1}{\Gamma(\alpha+1)} \cdot \phi(\|x\|,\|x\|)\left(m\left(t_{n}\right)-m(a)\right)^{\alpha},
\end{aligned}
$$

letting $n \rightarrow \infty$ in the above inequality and using the continuity of $m$, we get

$$
\lim _{n \rightarrow \infty}(U x)\left(t_{n}\right)=(U x)(a)=0,
$$

which prove the continuity of $U x$ at point $a$. Now let $t \in\left(a, T_{1}\right]$ be fixed and $\left\{t_{n}\right\}$ be a sequence in $\left(a, T_{1}\right]$ such that $t_{n} \rightarrow t$ as $n \rightarrow \infty$. With out restrictions we suppose that $t_{n} \geq t$ for $n$ large enough. We have

$$
\begin{aligned}
& (U x)\left(t_{n}\right)-(U x)(t) \mid \\
= & \frac{1}{\Gamma(\alpha)}\left|\int_{a}^{t_{n}} \frac{m^{\prime}(s) u\left(t_{n}, s, x(s), x(\lambda s)\right)}{\left(m\left(t_{n}\right)-m(s)\right)^{1-\alpha}} d s-\int_{a}^{t} \frac{m^{\prime}(s) u(t, s, x(s), x(\lambda s))}{(m(t)-m(s))^{1-\alpha}} d s\right| \\
\leq & \frac{1}{\Gamma(\alpha)} \mid \int_{a}^{t} \frac{m^{\prime}(s) u\left(t_{n}, s, x(s), x(\lambda s)\right)}{\left(m\left(t_{n}\right)-m(s)\right)^{1-\alpha}}-\frac{m^{\prime}(s) u(t, s, x(s), x(\lambda s))}{\left.(m(t)-m(s))^{1-\alpha}\right) d s \mid} \\
+ & \frac{1}{\Gamma(\alpha)}\left|\int_{t}^{t_{n}} \frac{m^{\prime}(s) u\left(t_{n}, s, x(s), x(\lambda s)\right)}{\left(m\left(t_{n}\right)-m(s)\right)^{1-\alpha}} d s\right| \\
\leq & \frac{1}{\Gamma(\alpha)} \int_{a}^{t}\left(\frac{m^{\prime}(s) \phi(|x|,|\lambda x|)}{\left(m\left(t_{n}\right)-m(s)\right)^{1-\alpha}}-\frac{m^{\prime}(s) \phi(|x|,|\lambda x|)}{\left.(m(t)-m(s))^{1-\alpha}\right) d s}\right. \\
+ & \frac{1}{\Gamma(\alpha)} \int_{t}^{t_{n}} \frac{m^{\prime}(s) \phi(|x|,|\lambda x|)}{\left(m\left(t_{n}\right)-m(s)\right)^{1-\alpha}} d s \\
\leq & \frac{1}{\Gamma(\alpha)} \phi\left(\|x\|,\|x\| \int_{a}^{t}\left(\frac{m^{\prime}(s)}{(m(t)-m(s))^{1-\alpha}}-\frac{m^{\prime}(s)}{\left.\left(m\left(t_{n}\right)-m(s)\right)^{1-\alpha}\right) d s}\right.\right. \\
+ & \frac{1}{\Gamma(\alpha)} \phi(\|x\|,\|x\|) \mid \int_{t}^{t_{n}} \frac{m^{\prime}(s)}{\left(m\left(t_{n}\right)-m(s)\right)^{1-\alpha}} d s \\
\leq & \frac{1}{\alpha \Gamma(\alpha)} \phi(\|x\|,\|x\|)\left[\left(m(t)-m^{\prime}(a)\right)^{\alpha}+\left(m\left(t_{n}\right)-m^{\prime}(t)\right)^{\alpha}-\left(m\left(t_{n}\right)-m(a)\right)^{\alpha}\right] \\
+ & \frac{1}{\alpha \Gamma(\alpha)} \phi(\|x\|,|\lambda|\|x\|)\left[\left(m\left(t_{n}\right)-m(t)\right)^{\alpha}\right.
\end{aligned}
$$

since $m$ is continuous function in $\left[a, T_{1}\right]$, then we hae $\lim _{n \rightarrow \infty}\left|(U x)\left(t_{n}\right)-(U x)(t)\right|=0$. Then $U x$ is continuous at $t$, then as consequence, $U x \in E$ for all $x \in E$ and $F: E \rightarrow E$ is well defined. For arbitrary fixed $x \in E$ and $t \in\left[a, T_{1}\right]$ we have

$$
\begin{aligned}
|(F x)(t)| & \leq \mid\left(f(t)|+| g(t, x(t)) \mid+\frac{|(T x)(t)|}{\Gamma(\alpha)} \int_{a}^{t} \frac{m^{\prime}(s)|u(t, s, x(s), x(\lambda x))|}{(m(t)-m(s))^{1-\alpha}} d s\right. \\
& \leq|f(t)|+\phi_{1}(|x(t)|)+g^{*}+\frac{(a+b\|x\|)}{\alpha \Gamma(\alpha)} \phi(\|x\|,|\lambda| \cdot\|x\|)\left(m\left(T_{1}\right)-m(a)\right)^{\alpha} .
\end{aligned}
$$

Then

$$
\|F x\| \leq\|f\|+\phi_{1}(\|x\|)+g^{*}+\frac{(a+b\|x\|)}{\Gamma(\alpha+1)} \phi(\|x\|,\|x\|)\left(m\left(T_{1}\right)-m(a)\right)^{\alpha},
$$


From assumption $\left(a_{9}\right)$, the fact that the functions $\phi_{1}, \phi$ are nondecreasing, and the above inequality, we infer that the operator $F$ maps $\overline{B\left(0, r_{0}\right)}$ into itself, where

$$
\overline{B\left(0, r_{0}\right)}=\left\{x \in E:\|x\| \leq r_{0}\right\}
$$

Now we will prove that the operator $F: \overline{B\left(0, r_{0}\right)} \rightarrow \overline{B\left(0, r_{0}\right)}$ is continuous, in order to prove this, it is sufficient to show that $f, g, T, U$ are continuous on $\overline{B\left(0, r_{0}\right)}$. From assumption $\left(a_{1}\right)$ the function $f$ is continuous. Firstly we show that $g$ is continuous on $\overline{B\left(0, r_{0}\right)}$. To do this, $\operatorname{let}\left\{x_{n}\right\} \subset \overline{B\left(0, r_{0}\right)}$ and $x \in \overline{B\left(0, r_{0}\right)}$ such that $\left\|x_{n}-x\right\| \rightarrow 0$ as $n \rightarrow \infty$, and we want to prove that $\left\|g x_{n}-g x\right\| \rightarrow 0$ as $n \rightarrow \infty$. In fact, for all $t \in[a, T]$, we have

$$
\begin{aligned}
\left|g\left(t, x_{n}(t)\right)-g(t, x(t))\right| & \leq \phi_{1}\left(\left|x_{n}-x\right|\right) \\
& \leq \phi_{1}\left(\left\|x_{n}-x\right\|\right)
\end{aligned}
$$

Then

$$
\left\|g\left(x_{n}\right)-g(x)\right\| \leq \phi_{1}\left(\left\|x_{n}-x\right\|\right), \quad n \in \mathbb{N} .
$$

Letting $n \rightarrow \infty$ in the above inequality, using the continuity of the function $\phi_{1}$, and the fact that $\phi_{1}(0)=0$, we get

$$
\lim _{n \rightarrow \infty}\left\|g\left(x_{n}\right)-g(x)\right\| \leq \phi_{1}\left(\lim _{n \rightarrow \infty}\left\|x_{n}-x\right\|\right)=\phi_{1}(0)=0 .
$$

Next,for the continuity of $U$ on $\overline{B\left(0, r_{0}\right)}$. To do this, we fix a real number $\rho>0$ and take arbitrary functions $x, y \in \overline{B\left(0, r_{0}\right)}$ such that $\|x-y\| \leq \rho$. For all $t \in\left[a, T_{1}\right]$, we have

$$
\begin{aligned}
|(U x)(t)-(U y)(t)| & \leq \frac{1}{\Gamma(\alpha)} \int_{a}^{t} \frac{m^{\prime}(s)|u(t, s, x(s), x(\lambda s))-u(t, s, y(s), y(\lambda s))|}{(m(t)-m(s))^{1-\alpha}} d s \\
& \leq \frac{\beta_{u}(\rho)(m(t)-m(a))^{\alpha}}{\alpha \Gamma(\alpha)} \\
& \leq \frac{\beta_{u}(\rho)\left(m\left(T_{1}\right)-m(a)\right)^{\alpha}}{\Gamma(\alpha+1)},
\end{aligned}
$$

where

$$
\begin{gathered}
\beta_{u}(\rho)=\sup \left\{\left|u\left(t, s, x_{2}, y_{2}\right)-u\left(t, s, x_{1}, y_{1}\right)\right|: t, s \in I, x_{1}, x_{2}, y_{1}, y_{2} \in\left[0, r_{0}\right]\right\} \\
\left|x_{2}-x_{1}\right| \leq \rho,\left|y_{2}-y_{1}\right| \leq \rho
\end{gathered}
$$

Thus,

$$
\|U x-U y\| \leq \frac{\beta_{u}(\rho)\left(m\left(T_{1}\right)-m(a)\right)^{\alpha}}{\Gamma(\alpha+1)} .
$$

By the uniform continuity of the function $u$ on $I \times I \times\left[0, r_{0}\right] \times\left[0, r_{0}\right]$ we have that $\beta_{u}(\rho) \rightarrow 0$ as $\rho^{+} \rightarrow 0$ and, therefore,

$$
\lim _{\rho^{+} \rightarrow 0}\|U x-U y\|=0
$$

Thus $U$ is continuous on $\overline{B\left(0, r_{0}\right)}$, and consequently, $F$ is continuous. Now let us take a nonempty set $W \subset \overline{B\left(0, r_{0}\right)}$. Let $\epsilon>0$ be fixed, choose $x \in W$ and $t_{1}, t_{2} \in I$ such that $\left|t_{2}-t_{1}\right| \leq \epsilon$. without loss of generality, we assume $t_{2} \geq t_{1}$. Then, we obtain

$$
\begin{aligned}
\mid & (U x)\left(t_{2}\right)-(U x)\left(t_{1}\right) \mid \\
= & \frac{1}{\Gamma(\alpha)}\left|\int_{a}^{t_{2}} \frac{m^{\prime}(s) u\left(t_{2}, s, x(s), x(\lambda s)\right)}{\left(m\left(t_{2}\right)-m(s)\right)^{1-\alpha}} d s-\int_{a}^{t_{1}} \frac{m^{\prime}(s) u\left(t_{1}, s, x(s), x(\lambda s)\right)}{\left(m\left(t_{1}\right)-m(s)\right)^{1-\alpha}} d s\right|
\end{aligned}
$$




$$
\begin{aligned}
& \leq \frac{1}{\Gamma(\alpha)}\left|\int_{a}^{t_{2}} \frac{m^{\prime}(s) u\left(t_{2}, s, x(s), x(\lambda s)\right)}{\left(m\left(t_{2}\right)-m(s)\right)^{1-\alpha}} d s-\int_{a}^{t_{2}} \frac{m^{\prime}(s) u\left(t_{1}, s, x(s), x(\lambda s)\right)}{\left(m\left(t_{2}\right)-m(s)\right)^{1-\alpha}} d s\right| \\
& +\frac{1}{\Gamma(\alpha)}\left|\int_{a}^{t_{2}} \frac{m^{\prime}(s) u\left(t_{1}, s, x(s), x(\lambda s)\right)}{\left(m\left(t_{2}\right)-m(s)\right)^{1-\alpha}} d s-\int_{a}^{t_{1}} \frac{m^{\prime}(s) u\left(t_{1}, s, x(s), x(\lambda s)\right)}{\left(m\left(t_{2}\right)-m(s)\right)^{1-\alpha}} d s\right| \\
& +\frac{1}{\Gamma(\alpha)}\left|\int_{a}^{t_{1}} \frac{m^{\prime}(s) u\left(t_{1}, s, x(s), x(\lambda s)\right)}{\left(m\left(t_{2}\right)-m(s)\right)^{1-\alpha}} d s-\int_{a}^{t_{1}} \frac{m^{\prime}(s) u\left(t_{1}, s, x(s), x(\lambda s)\right)}{\left(m\left(t_{1}\right)-m(s)\right)^{\alpha-1}} d s\right| \\
& \leq \frac{1}{\Gamma(\alpha)} \int_{a}^{t_{2}} m^{\prime}(s)\left|u\left(t_{2}, s, x(s), x(\lambda s)\right)-u\left(t_{1}, s, x(s), x(\lambda s)\right)\right|\left(m\left(t_{2}\right)-m(s)\right)^{\alpha-1} d s \\
& +\frac{1}{\Gamma(\alpha)} \int_{t_{1}}^{t_{2}} m^{\prime}(s)\left|u\left(t_{1}, s, x(s), x(\lambda s)\right)\right|\left(m\left(t_{2}\right)-m(s)\right)^{\alpha-1} d s \\
& +\frac{1}{\Gamma(\alpha)} \int_{a}^{t_{1}} m^{\prime}(s)\left|u\left(t_{1}, s, x(s), x(\lambda s)\right)\right|\left(\left(m\left(t_{2}\right)-m(s)\right)^{\alpha-1}-\left(m\left(t_{1}\right)-m(s)\right)^{\alpha-1}\right) d s \\
& \leq \frac{1}{\Gamma(\alpha)} \omega_{u}(\epsilon) \int_{a}^{t_{2}} m^{\prime}(s)\left(m\left(t_{2}\right)-m(s)\right)^{\alpha-1} d s \\
& +\frac{1}{\Gamma(\alpha)} \phi(\|x\|,\|x\|) \int_{t_{1}}^{t_{2}} m^{\prime}(s)\left(m\left(t_{2}\right)-m(s)\right)^{\alpha-1} d s \\
& +\frac{1}{\Gamma(\alpha)} \phi(\|x\|,\|x\|) \int_{a}^{t_{1}}\left(\left(m\left(t_{2}\right)-m(s)\right)^{\alpha-1}-\left(m\left(t_{1}\right)-m(s)\right)^{\alpha-1}\right) d s \\
& \leq \frac{\omega_{u}(\epsilon)\left(m\left(t_{2}\right)-m(a)\right)^{\alpha}}{\alpha \Gamma(\alpha)}+\frac{\phi(\|x\|,\|x\|)\left(m\left(t_{2}\right)-m\left(t_{1}\right)\right)^{\alpha}}{\alpha \Gamma(\alpha)} \\
& +\frac{\phi(\|x\|,\|x\|)\left[-\left(m\left(t_{2}\right)-m\left(t_{1}\right)\right)^{\alpha}+\left(m\left(t_{2}\right)-m(a)\right)^{\alpha}-\left(m\left(t_{1}\right)-m(a)\right)^{\alpha}\right.}{\alpha \Gamma(\alpha)} \\
& \leq \frac{\omega_{u}(\epsilon)\left(m\left(t_{2}\right)-m(a)\right)^{\alpha}}{\Gamma(\alpha+1)}+\frac{\phi(\|x\|,\|x\|)\left[\left(m\left(t_{2}\right)-m(a)\right)^{\alpha}-\left(m\left(t_{1}\right)-m(a)\right)^{\alpha}\right]}{\Gamma(\alpha+1)} \\
& \leq \frac{\omega_{u}(\epsilon)\left(m\left(t_{2}\right)-m(a)\right)^{\alpha}}{\Gamma(\alpha+1)} .
\end{aligned}
$$

Then in view of our assumptions we have

$$
\begin{array}{ll}
\mid & (F x)\left(t_{2}\right)-(F x)\left(t_{1}\right)|\leq| f\left(t_{2}\right)-f\left(t_{1}\right)|+| g\left(t_{2}, x\left(t_{2}\right)\right)-g\left(t_{1}, x\left(t_{1}\right)\right) \mid \\
+ & \frac{\left|(A x)\left(t_{2}\right)(U x)\left(t_{2}\right)-(A x)\left(t_{2}\right)(U x)\left(t_{1}\right)\right|}{\Gamma(\alpha)}+\frac{\left|(A x)\left(t_{2}\right)(U x)\left(t_{1}\right)-(A x)\left(t_{1}\right)(U x)\left(t_{1}\right)\right|}{\Gamma(\alpha)} \\
\leq & \left|f\left(t_{2}\right)-f\left(t_{1}\right)\right|+\left|g\left(t_{2}, x\left(t_{2}\right)\right)-g\left(t_{1}, x\left(t_{1}\right)\right)\right| \\
+ & \frac{\mid\left(A x\left(t_{2}\right) \|(U x)\left(t_{2}\right)-(U x)\left(t_{1}\right) \mid\right.}{\Gamma(\alpha)} \\
+ & \frac{\left|(A x)\left(t_{2}\right)-(A x)\left(t_{1}\right)\right|\left|(U x)\left(t_{1}\right)\right|}{\Gamma(\alpha)} \\
\leq & \left|f\left(t_{2}\right)-f\left(t_{1}\right)\right|+\left|g\left(t_{2}, x\left(t_{2}\right)\right)-g\left(t_{2}, x\left(t_{1}\right)\right)\right| \\
+ & \mid g\left(t_{2}, x\left(t_{1}\right)-g\left(t_{1}, x\left(t_{1}\right)\right) \mid+\frac{(a+b\|x\|)\left|(U x)\left(t_{2}\right)-(U x)\left(t_{1}\right)\right|}{\Gamma(\alpha)}\right. \\
+ & \frac{\left|(A x)\left(t_{2}\right)-(A x)\left(t_{1}\right) \|(U x)\left(t_{1}\right)\right|}{\Gamma(\alpha)} \\
\leq & \left|f\left(t_{2}\right)-f\left(t_{1}\right)\right|+\phi\left(\left|x\left(t_{2}\right)-x\left(t_{1}\right)\right|\right) \\
+ & \mid g\left(t_{2}, x\left(t_{1}\right)-g\left(t_{1}, x\left(t_{1}\right)\right) \mid\right. \\
+ & \frac{(a+b\|x\|)\left[\omega_{u}(\epsilon)\left(m\left(t_{2}\right)-m(a)\right)^{\alpha}+\phi(\|x\|,\|x\|)\left[2\left(m\left(t_{2}\right)-m\left(t_{1}\right)\right)^{\alpha}\right]\right]}{\alpha \Gamma(\alpha)} \\
+ & \frac{\mid\left(A x\left(t_{2}\right)-(A x)\left(t_{1}\right) \mid \phi(\|x\|,\|x\|)\left(m\left(t_{1}\right)-m(a)\right)^{\alpha}\right.}{\alpha \Gamma(\alpha)}
\end{array}
$$




$$
\begin{aligned}
& \leq \quad \omega(f, \epsilon)+\phi(\omega(x, \epsilon))+\omega_{g}\left(r_{0}, \epsilon\right) \\
& +\quad \frac{\left(a+b r_{0}\right)\left[\omega_{u}(\epsilon)\left(m\left(t_{2}\right)-m(a)\right)^{\alpha}+2 \phi\left(r_{0}, r_{0}\right)[\omega(m, \epsilon)]^{\alpha}\right]}{\Gamma(\alpha+1)} \\
& +\quad \frac{\omega(A x, \epsilon) \phi\left(r_{0}, r_{0}\right)\left(m\left(t_{1}\right)-m(a)\right)^{\alpha}}{\Gamma(\alpha+1)} \\
& \leq \quad \omega(f, \epsilon)+\phi(\boldsymbol{\Omega}(W, \epsilon))+\omega_{g}\left(r_{0}, \epsilon\right) \\
& +\quad \frac{\left(a+b r_{0}\right)\left[\omega_{u}(\epsilon)\left(m\left(t_{2}\right)-m(a)\right)^{\alpha}\right]}{\Gamma(\alpha+1)}+\frac{\omega(A x, \epsilon) \phi\left(r_{0}, r_{0}\right)\left(m\left(t_{1}\right)-m(a)\right)^{\alpha}}{\Gamma(\alpha+1)} \\
& \quad \leq \quad \omega(f, \epsilon)+\phi(\boldsymbol{\Omega}(W, \epsilon))+\omega_{g}\left(r_{0}, \epsilon\right) \\
& \quad+\quad \frac{\left(a+b r_{0}\right)\left[\omega_{u}(\epsilon)\left(m\left(T_{1}\right)-m(a)\right)^{\alpha}+2 \phi\left(r_{0}, r_{0}\right)[\omega(m, \epsilon)]^{\alpha}\right]}{\Gamma(\alpha+1)} \\
& \quad+\quad \frac{\omega(A x, \epsilon) \phi\left(r_{0}, r_{0}\right)\left(m\left(T_{1}\right)-m(a)\right)^{\alpha}}{\Gamma(\alpha+1)}
\end{aligned}
$$

Therefore,

$$
\begin{aligned}
\omega(F x, \epsilon) & \leq \omega(f, \epsilon)+\phi(\Omega(W, \epsilon))+\omega_{g}\left(r_{0}, \epsilon\right) \\
& +\frac{\left(a+b r_{0}\right)\left[\omega_{u}(\epsilon)\left(m\left(T_{1}\right)-m(a)\right)^{\alpha}+2 \phi\left(r_{0}, r_{0}\right)[\omega(m, \epsilon)]^{\alpha}\right]}{\Gamma(\alpha+1)} \\
& +\frac{\omega(A x, \epsilon) \phi\left(r_{0}, r_{0}\right)\left(m\left(T_{1}\right)-m(a)\right)^{\alpha}}{\Gamma(\alpha+1)}
\end{aligned}
$$

Let $\epsilon \rightarrow 0^{+}$, we obtain

$$
\mu(F W) \leq \phi(\mu(W))+\frac{\mu(A W) \phi\left(r_{0}, r_{0}\right)\left(m\left(T_{1}\right)-m(a)\right)^{\alpha}}{\Gamma(\alpha+1)}
$$

Taking in consideration assumption $\left(a_{3}\right)-($ iii $)$, we get

$$
\begin{aligned}
\mu(F W) & \leq \delta \theta(\mu(W))+\frac{q \mu(W) \phi\left(r_{0}, r_{0}\right)\left(m\left(t_{1}\right)-m(a)\right)^{\alpha}}{\Gamma(\alpha+1)} \\
& \leq\left(\delta+\frac{q \phi\left(r_{0}, r_{0}\right)\left(m\left(T_{1}\right)-m(a)\right)^{\alpha}}{\Gamma(\alpha+1)}\right) \mathrm{T}_{\max }(\theta, I)(\mu(W)) \\
& =\mathrm{T}\left(\lambda, \mathrm{T}_{\max }(\theta, I)\right)(\mu(W)),
\end{aligned}
$$

where

$$
\lambda=\left(\delta+\frac{q \phi\left(r_{0}, r_{0}\right)\left(m\left(T_{1}\right)-m(a)\right)^{\alpha}}{\Gamma(\alpha+1)}\right) .
$$

Since $\lambda \in[0,1]\left(\operatorname{see}\left(a_{9}\right)\right)$, from Lemma 2.3, $\mathrm{T}\left(\lambda, \mathrm{T}_{\max }(\theta, I)\right) \in \Phi$. Finally, by Lemma2.2, the operator $F$ has at least one fixed point $x^{*} \in B\left(0, r_{0}\right)$, which is the solution to the Eq. (1) satisfying $\left\|x^{*}\right\| \leq r_{0}$.

\section{Example}

Consider the integral equation

$$
x(t)=t^{3}+\frac{e^{-1} t}{8}+\frac{1}{4 \Gamma\left(\frac{1}{2}\right)} \int_{0}^{t} \frac{t s x(s) x(\lambda s)}{\sqrt{t-s}} d s, \quad t \in[0,1]
$$

In this example we have $a=0, T_{1}=1, \alpha=\frac{1}{2}, f(t)=t^{3}, t \in[0,1]$ and this function satisfies assumption $\left(a_{1}\right)$ with $\|f\|=1$. Here

$$
g(t, x(t))=\frac{e^{-1} t}{8}, \quad(t, x) \in[0,1] \times \mathbb{R},
$$


satisfies assumption $\left(a_{2}\right)$ with $\phi_{1}(t)=\theta(t)=\delta=0$. Moreover, in this case we have $g^{*}=\frac{e^{-1}}{8}(A x)(t)=\frac{1}{4}$, satisfies assumption $\left(a_{5}\right)$ with $a=\frac{1}{4}, b=0, q=0$. Also, $u(t, s, x, y)=t s x y$, satisfies assumption $\left(a_{7}\right)$ with $\phi(x, y)=x y$. Moreover, $m(t)=t$, satisfies assumption $\left(a_{8}\right)$

$$
\begin{gathered}
\|f\|+\phi_{1}\left(r_{0}\right)+g^{*}+\frac{\left(a+b r_{0}\right)}{\alpha \Gamma(\alpha)} \phi\left(r_{0}, r_{0}\right)\left(m\left(T_{1}\right)-m(a)\right)^{\alpha} \leq r_{0} \\
1+\frac{e^{-1}}{8}+\frac{1}{2 \Gamma\left(\frac{1}{2}\right)} \cdot r_{0}^{2} \leq r_{0}
\end{gathered}
$$

then

$$
r_{0}=\Gamma\left(\frac{1}{2}\right)\left(\sqrt{\Gamma\left(\frac{1}{2}\right)} \pm \sqrt{\Gamma\left(\frac{1}{2}\right)-2\left(1+\frac{e^{-1}}{8}\right)}\right)
$$

Data Availability (excluding Review articles)

Applicable.

\section{References}

[1] A. Aghajani, J. Banaś, N. Sabzali, Some generalizations of Darbo fixed point therom and applications, Bull. Belg. Math. Soc. simon Stevin. 20(2)(2013), 345-358. https://doi.org/10.36045/bbms/1369316549

[2] A. Aghajani, J. Banaś, Y. Jalilian, Existence of solutions for a class of nonlinear Volterra singular integral equations, Comput. Math. Appl. 62(2011), 1215-1227. https://doi.org/10.1016/j.camwa.2011.03.049

[3] A. Aghajani, R. Allahyari, M. Mursaleen, Ageneralization of Darbo's theorm with application to the solvability of systems of integral equations, J. Comput. Appl. Math. 260(2014), 68-77.https://doi.org/10.1016/j.cam.2013.09.039

[4] D. Beglov, B. Roux, An integral equation to describe the solvation of polar molecules in liquid water, J. Phys. Chem. B. 101(1997), 7821-7826. https://doi.org/10.1021/jp971083h

[5] G. Darbo, Punti untiti in transformazioni a condominio noncompatto, Rend. Sem. Mat. Univ. Padora 24 (1955), 84-92.http://www. numdam. org

[6] H.W. Hethcote, D.W. Tudor, Integral equation models for endemic infectious diseases, J. Math. Biology. 9(1980), 37-47, doi:10.1007/BF00276034

[7] HA. Schenck, Improved integral formulation for acoustic radiation problem, J Acoust Soc Am. 44 (1968), 41-58. https://doi.org/10.1121/1.1911085

[8] I.M. Olaru, Generalization of an integral equation related to some epidemic models, Carpathian J. Math. 26(1)(2010), 92-96.http://www.jstor.org/stable143999436

[9] J. Banaś and A. Martinon, Monotonic solutions of quadratic integral equation of voltera type, Comput. Math. Appl. 47(2004), 271-279. https://doi.org/10.1016/S0898-1221(04)90024-7

[10] J. Banaś and B. Rzepka, Monotonic solutions of quadratic integral equation of fractional order, J. Math. Anal. Appl. 332(2007), 1370-1378.

[11] J. Banas and K. Goebel, Measures of Noncompactness in Banach Spaces, Lect. Notes in Math. 60, M. Dekker, New york and Basel, 1980. 
[12] J. Banaś, M. Lecko and w. G. El-sayed, Existence theorem of some quadratic integral equations, J. Math. Anal. Appl. 222(1998), 276-285. https://doi.org/10.1006/jmaa.1998.5941

[13] J. Appell, P.P. Zabrejko, Nonlinear Superposition operator, Cambridge Tracts in Mathematics.95,Cambridge University Press, 1990. https://doi.org/10.1017/CBO9780511897450

[14] J. Sladek, V. Sladek, S.N. Atluri, Local boundary integral equation(LBIE)method for solving problem of elasticity with nonhomogeneous material properties, Computational Mechanics. 24(2000), 456-462. https://doi.org/10.1007/s004660050005

[15] K. Sadarangani, B. Samet, Solvability of a fractional integral equation with the concept of measure of noncompactness, Bull. Belg. Math. Soc. Simon Stevin. 24 (2017), 1-17. https://doi.org/10.36045/bbms/1489888812

[16] M.A. Darwish, On monotonic solutions of a singular quadratic integral equation with supremum,Dynam. Systems appl. 17(20008), 539-550.

[17] M.A. Darwish, On quadratic integral equation of fractional orders,J. Math. Anal. Appl. 311(2005), $112-119$. https://doi.org/10.1016/j.jmaa.2005.02.012

[18] M.A. Darwish, On Erdélyi -Kober fractional Urysohn-Volterra quadratic integral equation,Appl. Math. Comput.273(2016), 562-569. https://doi.org/10.1016/j.amc.2015.10.040

[19] M.A. Darwish, K. Sadarangani, On quadratic integral equation with supremum involving Erdélyi -Kober fractional order, Mathematische Nachrichten. 288(5-6)(2015),566-576.

[20] M. Benchohra, D. Seba, Integral equations of fractional order with multiple time delays in banach spaces,Electron. J. differential Equations. 65(2012), 1-8.

[21] M. Cichon, M.A. Metwali, On quadratic integral equations in Orlicz spaces,j. Math. Anal. Appl. 387(2012), 419-432. https://doi.org/10.1016/j.jmaa.2011.09.013

[22] R. Agarwal, M. Meehan, D. O'Regan, Fixed point Theory and Applications, Cambridge University Press, 2004.

[23] R.P. Agarwal, B. Samet, An existence result for a class of nonlinear integral equations of fractional orders, Nonlinear Analysis. Modelling and Control. 21(5)(2016), 616- 629. https://doi.org/10.15388/NA.2016.5.10

[24] R. Hilfer, Applications of Fractional Calculus in Physics, World Scientific. Singapore, 2000. https://doi.org/10.1142/3779

[25] S. Chandraseker, Relative transfer, Dover publications, Newyork, 1960.

[26] S.Hu, M. Khavani and W. Zhuang, Integral equations arising in the kinetic theory of gases, Appl. Anal. 34(1989), 261-266. https://doi.org/10.1080/00036818908839899

[27] U. Çakan, I. Ozdemir, An application of Darbo fixed-point theorem to a class of functional integral equations, Numer. Funct. Anal. Optim. 36(2015), 29-40. https://doi.org/10.1080/01630563.2014.951771

[28] V. Muresan, Volterra integral equations with iterations of linear modification of argument, Novi Sad J. Math. $33(2003), 1-10$.

[29] W.G. El-Sayed, B. Rzepka, Nondecreasing solutions of a quadratic integral equation of Urysohn type, Comput. Math. Appl. 51 (2006), 1065-1074. https://doi.org/10.1016/j.camwa.2005.08.033 


\section{Supplementary Materials}

Not applicable.

\section{Conflicts of Interest}

The authors declare that they have no competing interests. Funding Statement

The research was self-sposored by the author.

\section{Acknowledgments}

The authors like to thank the anonymous referees for their valuable comments and suggestions, which greatly improved the presentation of this paper. 